\title{
Reply to Dlouha et al
}

European Journal of Human Genetics (2010) 18, 1275; doi:10.1038/ejhg.2010.123; published online 28 July 2010

We appreciate the opportunity to reply to the letter by Dlouha $\mathrm{et} \mathrm{al,}{ }^{1}$ who have attempted to replicate the association of the FTO variant rs17818902 with obesity that we have recently identified in a selfcontained population of Sorbs from Germany. ${ }^{2}$ To test the SNP in a population closely related to the Sorbs, the authors genotyped this polymorphism in a Czech-Slavonic population. They failed to replicate the initial association and postulated that gene-gene and/or gene-environmental interactions might explain variability in the exact role of this variant in the pathogenesis of obesity in different populations.

We agree that a different definition of the investigated phenotype (BMI) is unlikely to explain the observed discrepancy between Czechs and Sorbs. Even though no major differences in genetic background between two neighbouring middle-European Slavonic populations can be assumed ${ }^{3}$ recent studies provide strong evidence that population structure represents a well-recognized challenge for disease-association studies, and that spurious associations can arise if genetic structure is not adequately accounted for. ${ }^{4}$ So far, there is no straightforward explanation for different effect sizes, or even effect directions, of recently identified susceptibility loci in diverse populations (also if ethnic background is comparable). Even if the combined effect in a meta-analysis strongly supports a genetic association the variant does not show replicable effects in all investigated samples. This cannot be explained exclusively by sample size, allele frequencies or phenotypic distribution.

As geographic origin of a sample has to be considered when evaluating genome-wide association studies among Europeans, ${ }^{4}$ the same may be true when attempting to replicate initial association findings. Using principal component analysis we are currently evaluating the genetic variation between Slavonic populations (Sorbs, Czechs, and Poles) and Germans. Our preliminary data indicate that the Sorbs were most closely related to the Poles, but measures such as Fst are summaries and do not necessarily capture all kinds of changes in a certain genetic region.

When we investigated the proportion of individuals with at least one other individual IBS at all SNPs across random genomic regions (methods based on Kong $e t a l^{5}$ ), we found that IBS sharing in the Sorbs was much greater compared with that in outbred populations, such as the controls from the WTCCC. ${ }^{6}$ The proportions of subjects who have at least one other individual with IBS $\geqslant 1$ at all SNPs across the chromosome 15-long and MHC region were 29.3 and $40.7 \%$, respectively, in the Sorbs compared with 0.8 and $10.2 \%$, respectively, in the WTCCC sample set.

We would not necessarily expect to see association at exactly the same SNP because of possible allelic heterogeneity. Moreover, linkage disequilibrium patterns are also likely to be different and, although we cannot provide a definitive explanation for the discrepancies observed, we believe that a unique population history of the Sorbs may contribute to these different associations.

Despite failing to replicate the findings of the Sorbian study in the present paper by Dlouha et al, ${ }^{1}$ it is noteworthy that the relevance of the third intron of FTO is supported by analyses of patterns of selection in the FTO region. These analyses show signatures of recent selection at the FTO locus in human populations, ${ }^{7}$ and the strongest signal is observed in the third intron, the region in which the strong association with BMI in the Sorbs was recently described. ${ }^{2}$ Resequencing studies will be required to further elucidate the functional variants behind the association signals.

Furthermore, the possible impact of factors such as epigenetics and the effects of rare variants not covered by the available assays should be considered. Last, but not the least, we are aware of the possibility that the finding in Sorbs may be false positive. Given that the finite size of the Sorbian population limited further internal replication, we encourage and appreciate replication attempts by independent groups such as the one reported by Dlouha et al. ${ }^{1}$

\section{CONFLICT OF INTEREST}

The authors declare no conflict of interest.

Anke Tönjes ${ }^{1}$, Peter Kovacs ${ }^{2}$, Katherine Elliott ${ }^{3}$, Eleftheria Zeggini ${ }^{4}$, Mark I McCarthy ${ }^{3,5}$ and Michael Stumvoll ${ }^{1}$

${ }^{1}$ Department of Medicine, University of Leipzig, Leipzig, Germany;

${ }^{2}$ Interdisciplinary Centre for Clinical Research, University of Leipzig, Leipzig, Germany;

${ }^{3}$ Wellcome Trust Centre for Human Genetics, University of Oxford, Oxford, UK;

${ }^{4}$ Wellcome Trust Sanger Institute, Cambridge, UK; ${ }^{5}$ Oxford Centre for Diabetes, Endocrinology and Metabolism, Oxford, UK

E-mail: Michael.Stumvoll@medizin.uni-leipzig.de

1 Dlouha D, Adamkova V, Lanska $V$ et al: Lack of association between a new tag SNP in the FTO gene and BMI in Czech Slavonic population. Eur J Hum Genet 2010, 18: 1274.

2 Tönjes A, Zeggini E, Kovacs P et al: Association of FTO variants with BMI and fat mass in the self-contained population of Sorbs in Germany. Eur J Hum Genet 2010; 18: 104-110.

3 Nelis M, Esko T, Mägi R et al: Genetic structure of Europeans: a view from the North-East. PLoS One 2009; 4: e5472.

4 Novembre J, Johnson T, Bryc K et al: Genes mirror geography within Europe. Nature 2008; 456: 98-101.

5 Kong A, Masson G, Frigge $M$ et al: Detection of sharing by descent, long-range phasing and haplotype imputation. Nat Genet 2010; 9: 1068-1075.

6 Wellcome Trust Case Control Consortium: Genome-wide association study of 14000 cases of seven common diseases and 3000 shared controls. Nature 2007; 447: 661-678.

7 Dietrich K, Schröck K, Schleinitz D et al: Signatures of selection at the FTO (Fat Mass and Obesity Associated) locus in human populations. Diabetologia 2009; 52: S48. 